\title{
Induction, Regulation, and Role in Pathogenesis of Appressoria in Monilinia fructicola
}

\author{
Miin-Huey Lee and Richard M. Bostock
}

Department of Plant Pathology, University of California, One Shields Ave., Davis 95616.

Current address of M.-H. Lee: Department of Plant Pathology, National Chung-Hsing University, No. 250 Kao-Kuang Rd., Taichung 402, Taiwan. Accepted for publication 1 May 2006.

\begin{abstract}
Lee, M.-H., and Bostock, R. M. 2006. Induction, regulation, and role in pathogenesis of appressoria in Monilinia fructicola. Phytopathology 96:1072-1080.

Monilinia fructicola, which causes brown rot in stone fruit, forms appressoria on plant and artificial surfaces. On nectarine, the frequency of appressoria produced by conidial germlings depends to a large degree on the stage of fruit development, with numerous appressoria formed on immature (stage II) nectarine fruit, and no appressoria observed on fully mature fruit (late stage III). On polystyrene surfaces, appressorium formation was increased from $<10 \%$ of germinated conidia to $>95 \%$ of

appears to be regulated by the topography of the plant surface. On fruit, appressoria formed on stomatal guard cell lips, on the grooves of lateral cells adjacent to stomata or between two epidermal cells, and on the convex surfaces of epidermal cells. Pharmacological effectors indicate that cyclic AMP-, MAP kinase-, and calcium/calmodulin-dependent signaling pathways are involved in the induction and development of appressoria. KN-93, an inhibitor of calmodulin-dependent protein kinase II, did not inhibit conidial germination but did inhibit appressorium formation and brown rot development on flower petals, suggesting that appressoria are required for full symptom development on Prunus spp. petals.
\end{abstract} germinated conidia when the conidia were washed to remove residual nutrients and self-inhibitors. $M$. fructicola appressorium formation also
Additional keywords: penetration, quiescent infections.
Brown rot disease caused by Monilinia spp. is a major disease of stone fruit throughout the world. In California, Monilinia fructicola (G. Wint.) Honey is the principal species responsible for fruit brown rot and blossom and twig blight in peach (Prunus persica (L.) Batsch) and nectarine (Prunus persica var. nectarina Maxim). Blossoms and mature fruit are the most susceptible host organs. However, quiescent infections that frequently occur on immature fruit (stage II fruit) can develop into pre- and postharvest brown rot, resulting in severe yield losses $(6,29)$. Relatively little is known about pathogenicity and penetration by this pathogen on different developmental stages of host tissues.

Phytopathogenic fungi utilize diverse mechanisms to infect host tissue, which include chemical and thigmotropic sensing to optimally position infection structures, the production of enzymes to degrade host surfaces, and the formation of specialized structures such as appressoria (28). Appressoria are required for penetration by important phytopathogenic fungi, such as Colletotrichum spp., Magnaporthe grisea, and many rust and powdery mildew fungi $(27,34)$. In Colletotrichum gloeosporioides, which has a wide host range, nongerminated and germinated appressoria have been suggested to function as quiescent structures in fruit such as unripe banana, avocado, grape, and blueberry $(11,30)$.

Several types of appressoria and appressorial induction mechanisms have been identified. Perhaps the most well-studied are the highly melanized appressoria from $M$. grisea and Colletotrichum spp., which can generate extremely high turgor pressures. The generation of high pressure is considered critical for these fungi

Corresponding author: R. M. Bostock; E-mail address: rmbostock@ucdavis.edu

* The $\boldsymbol{e}$-Xtra logo stands for "electronic extra" and indicates that the online version contains supplemental material not included in the print edition. Figures 1 to 4 appear in color online.

DOI: 10.1094/PHYTO-96-1072

(C) 2006 The American Phytopathological Society to penetrate thick cuticle layers, such as in rice leaves and avocado fruit. Many non- or lightly melanized appressoria from species such as the barley powdery mildew pathogen Blumeria graminis are able to directly penetrate the epicuticular layer (1). The appressoria of $B$. graminis generate lower pressure (2 to $4 \mathrm{Mpa})$ compared with the heavily melanized appressoria of $M$. grisea (up to $8 \mathrm{Mpa}$ ) (17,31). The combination of enzymatic degradation and mechanical force is suggested to contribute to penetration by B. graminis (31).

Appressorium formation is stimulated by a variety of environmental factors. Plant compounds, such as cutin monomers, wax, ethylene, and leaf volatiles, and surface characters, such as hydrophobicity and topography, induce appressorium formation (11, 12). Physical factors, including hydrophobicity and topography, are common appressorial inducers for many fungi. Hydrophobic surfaces, such as polystyrene, strongly induce appressorium formation in $M$. grisea (25) and stimulate appressorium formation in Uromyces appendiculatus (33), Phytophthora palmivora (5), and some fungal pathogens of insects (14). A number of appressorial fungi, such as $U$. appendiculatus (38), Cochliobolus sativus (8), C. graminicola (24), and P. palmivora (5), appear to respond thigmotropically to topographical signals.

Several important intracellular signaling pathways of appressorium formation have been investigated, including pathways involving $G$ protein-cyclic adenosine $3^{\prime}, 5^{\prime}$-cyclic monophosphate (cAMP)-, MAP kinase-, and calcium/calmodulin-mediated signaling. Pharmacological effectors and mutants in pathway-essential genes have been used in these investigations. In $M$. grisea, cAMP action and phosphorylation events catalyzed by MAP kinase coregulate appressorium formation (32). The cooperation of two pathways for appressorium formation also was found in $B$. graminis (23). In Colletotrichum trifolii, both cAMP and calcium/calmodulin regulate appressorium formation $(37,39)$, and MAP kinase action and calcium/calmodulin regulate appressorium formation of C. gloeosporioides (20,21). 
Understanding mechanisms associated with the infection process of Monilinia fructicola could provide insights for improved management of this worldwide problem. Production of simple appressoria by $M$. fructicola and subsequent penetration of host tissue have been reported previously on apricot fruit and flower petals of cherry and plum (9). However, in that study, the frequency of formation of simple appressoria was very low (1\% in vitro). In the present study, we examined appressorium formation on nectarine fruit and evaluated the importance of appressoria in brown rot disease development in Prunus petals and in different stages of nectarines. We also tested various external and internal chemical signals to gain an understanding of the regulation of appressorium formation in this species.

\section{MATERIALS AND METHODS}

Fungal and plant materials. A single-spore isolate of $M$. fructicola, isolate MUK-1, which was cultured from infected peach fruit from a local orchard in California, was used throughout this work (7). This isolate has been used routinely in our research for $>10$ years because of its stability in culture, high capacity for sporulation, and strong virulence on peach and other stone fruit. The fungus was routinely grown on V8 juice agar at room temperature for macroconidia production. Flower petals and fruit of Prunus spp., including almond (Prunus dulcis (Mill.) Webb), nectarine $(P$. persica var. nectarina), and peach $(P$. persica) were harvested from 6-year-old trees in an orchard on the Plant Pathology Department research farm at the University of California, Davis. The fruit developmental stage was defined according to Connor, based on fruit size and embryo development, as described by Zucconi (40). In stage I, the volume of the pericarp increases rapidly. In stage II, pericarp growth is slow but embryo development is rapid, with concomitant lignification of the endocarp (pit hardening). In stage III, the endocarp completes its development and the pericarp resumes a rapid increase in volume through cell expansion and division. In our experiments, fruit stages were determined by measuring the diameters of representative fruit and assessing embryo development by the ease of splitting the pit and embryo with a knife. Stage I fruit have a diameter $<2.8 \mathrm{~cm}$ and embryos and pits that are readily split. Stage II fruit have a diameter between 2.8 and $3.3 \mathrm{~cm}$ and a hardened endocarp (pit and embryo not cleaved with a knife). Stage III fruit have a diameter $>3.3 \mathrm{~cm}$.

Yellow onions (Allium cepa L.) were purchased from a local supermarket.

Spore washing and appressoria studies. To make spore suspensions, conidia were collected from 7- to 10-day-old V8 juice agar plates by flooding with sterile water, usually $5 \mathrm{ml}$ of water for one 9-cm plate. Conidial suspensions were filtered through one layer of Miracloth (Calbiochem Co., San Diego, CA) to remove mycelia. Conidia were washed with $1 \mathrm{ml}$ of water twice by centrifugation $(10,600 \times g$ for $30 \mathrm{~s})$ and resuspension. The original and the first washing supernatants were saved for other uses as described below. To evaluate appressorium formation, the washed or unwashed spore suspensions were diluted to $5 \times 10^{5}$ spores $/ \mathrm{ml}$ and mixed with other components as appropriate.

To study appressoria development in $M$. fructicola, we first established optimal conditions for conidial germination, because macroconidia germinate poorly $(<1 \%)$ in water. However, $1 \mathrm{mM}$ sucrose greatly increased germination of washed and unwashed conidia to $>95 \%$. In preliminary experiments, no notable differences in conidial germination, germ tube elongation, or appressorium formation were found when sucrose concentration was varied between 1 and $50 \mathrm{mM}$ over a 24-h incubation period at room temperature (data not shown). Thus, $1 \mathrm{mM}$ sucrose served as the basal solution for in vitro appressorial studies, with a spore density of 100 conidia/ $\mu$ l unless otherwise noted.
A standard assay involved placement of a 5- $\mu$ l drop on a polystyrene petri dish lid (cat. no. 08-757-12 and 08-757-13A; Fisher Scientific, Hampton, NH), followed by incubation in a moist chamber at room temperature. To study the effects on appressorium formation of supernatant washings and nutrients from clarified V8 (cV8) juice, conidia were suspended in a solution that contained a 1:5 dilution of supernatant washings or serially diluted cV8 juice broth. To study the effects of self-inhibitors, various amounts of solutions with equal concentrations of conidia and sucrose were placed on petri dish lids.

Appressorium formation on artificial surfaces and in planta. To test the effects of artificial surfaces on appressorium formation, a conidial suspension $(20 \mu \mathrm{l})$ was placed on a small petri dish lid, a clean glass slide, the hydrophobic side or hydrophilic side of gel bond film (FMC Co., Philadelphia, PA), or Parafilm ' $\mathrm{M}$ '. The gel bond film is a polyester sheet on which a hydrophilic surface is provided by a coating of agarose. Conidial germination and appressorium formation were examined by microscopy after 6 and $18 \mathrm{~h}$ of incubation. The glass slide (cat. no. 3011; Gold Seal, Erie Scientific Co., Portsmouth, NH) was cleaned by dipping into $95 \%$ ethanol overnight and rinsing thoroughly with distilled water. A 5- $\mu$ l unwashed spore suspension containing 250 or 500 spores without sucrose was inoculated onto fruit and petal surfaces. The inoculated areas were stained with lactophenolcotton blue and examined under the microscope. At least 20 specimens from more than five fruit of various stages of each Prunus spp. examined were obtained. Sucrose was absent from the conidial suspension when inoculated on plants except as indicated.

Pharmacological effectors. The pharmacological effectors used to evaluate the possible involvement of intracellular signaling pathways in $M$. fructicola appressorium formation are cAMP (Sigma Chemical, St. Louis); an inhibitor of cyclic nucleotide phosphodiesterase 3-isobutyl-1-methylxanthine (IBMX; Sigma); a calcium-calmodulin kinase inhibitor, KN-93 (Alexis Biochemicals, San Diego, CA); and a MAP kinase inhibitor, PD98059 (Tocris Cookson Ltd., Ellisville, MO). Cyclic AMP and KN-93 were dissolved in water as stock solutions and stored at $-20^{\circ} \mathrm{C}$. IBMX was dissolved in ethanol and stored at $-20^{\circ} \mathrm{C}$, and PD98059 was dissolved in dimethyl sulfoxide and stored at $23 \pm$ $2{ }^{\circ} \mathrm{C}$. If solvents were used to dissolve the effectors, then the solvent was added at the same concentration in the control treatment. A 5 - $\mu$ l drop of each solution containing conidia at the indicated concentrations was placed on small petri dish lids. Conidial germination and appressorium formation then were examined after 6- and 18-h incubation periods.

Inoculation of flower petals and onion. Onion epidermal layers frequently have been used to study appressorium formation in Magnaporthe grisea and are available throughout the year (2). Monilinia fructicola conidia were scraped from V8 juice agar cultures with a surgical blade without water to reduce the contamination of nutrients from the V8 juice medium. The collected spores were suspended in sterile water and adjusted to $1 \times 10^{5}$ or $5 \times 10^{4}$ spores $/ \mathrm{ml}$. The spore suspension $(5 \mu \mathrm{l})$ was applied to onion epidermal strips floating on water, and the inoculated strips then were incubated at $23 \pm 2{ }^{\circ} \mathrm{C}$. Fully expanded blossoms from almond, nectarine, and peach were collected from the orchard and the petals excised and placed in a moist chamber. Spore suspension $(5 \mu \mathrm{l})$ with or without KN-93 was inoculated onto each petal. Brown rot lesions that developed on the petals were photographed with a digital camera and the lesion size was measured using Spot image analysis software (Spot RT Software version 3.5.1; Diagnostic Instruments, Inc., Sterling Heights, MI).

Data analysis. Experiments were conducted with at least three replicates of each treatment within an experiment and all experiments were repeated at least once with similar results. The experimental design was a complete random design unless otherwise noted. Data were analyzed using the appropriate statistical tests as 
indicated in the figures and tables, which included paired $t$ test, regression analysis, and analysis of variance using Tukey's and Dunnett's tests as implemented in PC-SAS (SAS Institute, Cary, NC).

\section{RESULTS}

Appressorium formation in M. fructicola. M. fructicola readily produced appressoria on both artificial and plant surfaces (Fig. 1). Washed macroconidia differentiated into lightly pigmented or nonpigmented swollen structures (appressoria) on artificial surfaces. On the polystyrene surface, appressoria were oval shaped and formed immediately after conidia germinated, typically within 3 to $4 \mathrm{~h}$ of incubation. Some appressoria were also hook shaped (Fig. 1A, arrows). On flower petals, stage II nectarine fruit, and leaves, $M$. fructicola formed appressoria similar to those formed on polystyrene (Figs. 1C and D and 2).

The frequency of appressorium formation was related to the developmental stages of nectarine fruit (Fig. 2A to C). When fruit were $<1.8 \mathrm{~cm}$ in diameter (early stage I), conidia produced appressoria less frequently than on late stage I and stage II fruit (Fig. 2A). This was observed on all four nectarine cultivars examined in this study. In all, $\approx 80 \%$ of germinated conidia produced appressoria on larger fruit in late stage I $(2.5 \mathrm{~cm}$ in diameter; before pit hardening), and $>90 \%$ of germinated conidia formed appressoria on stage II fruit of cvs. Fantasia, Independence, and Dwarf (Fig. 2B). No appressoria formed on fully mature fruit (late stage III) of Fantasia (Fig. 2C). In the latter case, macroconidia germinated and penetrated through microscopic fissures that often are present on mature fruit surfaces (Fig. 2D).

Appressoria associated with penetration of the epicuticular layers of immature fruit were observed (Fig. 3A and B). The surfaces of appressorium penetration sites appeared to be depressed by force (Fig. 3A and B, arrows). M. fructicola using appressoria to penetrate petal epicuticular layers and onion epidermal layers were clearly evident (Fig. 3C to F). M. fructicola conidia germinated on petals within $2 \mathrm{~h}$ after inoculation. Most conidia

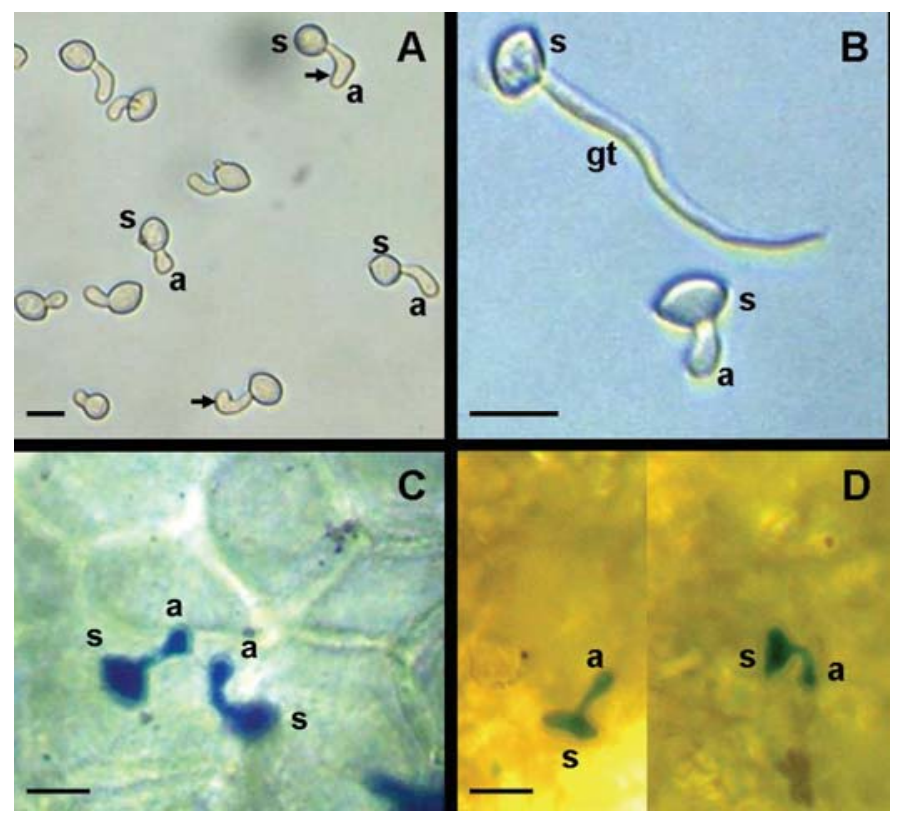

Fig. 1. Appressorium formation of Monilinia fructicola on artificial and plant surfaces. A and B, Conidia produce three types of appressoria on a polystyrene surface after $9 \mathrm{~h}$ incubation at $25^{\circ} \mathrm{C}$. A, Spores (s) with newly formed appressoria (a). The hook-shape is indicated by arrows. A and B, Conidium with a hyphal germ tube (gt), for comparison with a conidium with an appressorium. C and D, Similar type appressoria form on plant surfaces. C, Appressoria on stage II nectarine fruit of cv. Independence. D, Appressoria on a nectarine leaf of cv. Fantasia. Bar in each panel indicates $15 \mu \mathrm{m}$. formed appressoria at $4 \mathrm{~h}$ after inoculation. Conidia and appressoria located on the surfaces were stained with lactophenol-cotton blue. Infected plant cells became translucent at $9 \mathrm{~h}$ after inoculation, allowing penetration hyphae subtending the surface to be observed as colorless structures even after treatment of the tissue with the stain (Fig. 3C to E). The production of appressoria and penetration hyphae also was observed on onion epidermal layers at $4 \mathrm{~h}$ after inoculation using phase contrast microscopy. The fungal structures on the surface were darker than those which grew into and underneath the onion epidermal cells (Fig. 3F).

Appressorium formation is associated with surface topography. $M$. fructicola formed appressoria on stomatal guard cell lips or in between the stomata and their adjacent areas on stage II fruit (Fig. 4). Conidia primarily formed simple appressoria when they fell directly upon the stomata (Fig. 4A). In some instances, conidia iteratively produced two or three sequential appressorialike swellings on the stomatal lips (Fig. 3B). However, we did not find direct evidence that $M$. fructicola penetrates stomata using appressoria. In some cases, conidia germinated toward the lateral cells and produced appressoria at the cell junctions (Fig. 4C).

Appressoria often formed at the grooves of epidermal cell wall junctions when conidia lay on the fruit epicuticular surface (Figs. $1 \mathrm{C}$ and $4 \mathrm{D}$ to $\mathrm{F}$ ). This appressorium formation appeared in response to elevated structures (Fig. 4D to F). In some cases, appressoria formed on the convex surface of epidermal cells (Fig. 4G).

Factors involved in appressorium formation on a polystyrene surface. Conidia harvested directly from V8 juice agar plates usually produced few appressoria following germination on artificial surfaces (Table 1). In order to increase the frequency of appressorium formation, the washing procedure was performed as described previously. Washing the conidia markedly increased the frequency of appressoria formed from germinated conidia. Addition of the original supernatant to the washed conidia restored the

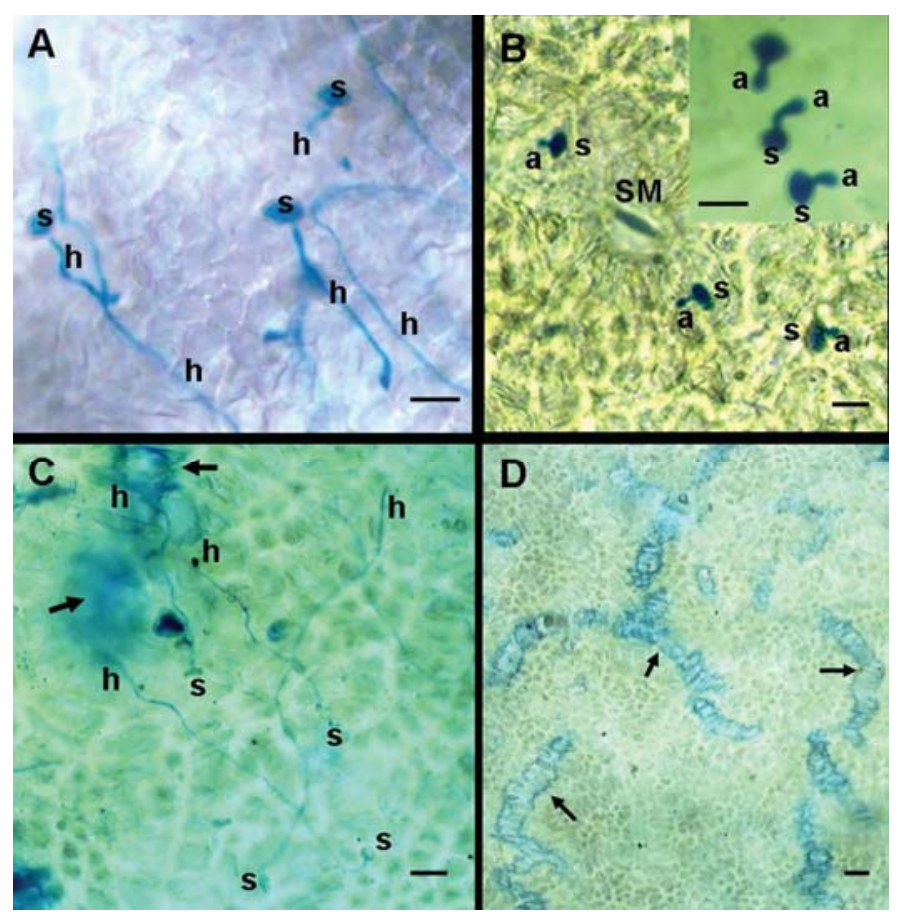

Fig. 2. Appressoria produced on different stages of nectarine fruit (cv. Fantasia) after $9 \mathrm{~h}$ incubation of Monilinia fructicola conidia at $25^{\circ} \mathrm{C}$. A, Early stage I fruit. Germinated spores (s) produce long hyphal germ tubes (h), some with swellings on the germ tube apices. B, Germinated conidia produce appressoria (a) on stage II fruit. Insert is a high magnification view. C, Hyphal germ tubes grow toward the fissures (arrows) on fully mature fruit. D, Microscopic fissures exist on fully mature fruit surfaces (arrows). Bar in each panel indicates $15 \mu \mathrm{m}$. SM, stoma. 
inhibition of appressorium development to that of the unwashed conidia, even after $16 \mathrm{~h}$ of incubation. The addition of the first washing supernatant did not significantly influence appressorium formation (data not shown). No difference in germination rate was observed among any of the treatments (Table 1).

Experiments were conducted to determine whether any components, such as self-inhibitors and nutrients, that might influence appressorium formation were removed or diluted during the wash procedure. To test the effect of nutrients on appressoria inhibition, clarified V8 juice broth was diluted serially and mixed with conidial suspension. The results showed that 25 and $95 \%$ of the washed conidia produced long germ tubes rather than appressoria after treatment with 0.1-fold dilution and full-strength $(1 \times) \mathrm{cV} 8$ juice broth, respectively, at $6 \mathrm{~h}$ (Table 2). However, it was observed in the $\mathrm{cV} 8$ juice broth treatments that two germination events from one conidium frequently occurred after $16 \mathrm{~h}$ of incu-

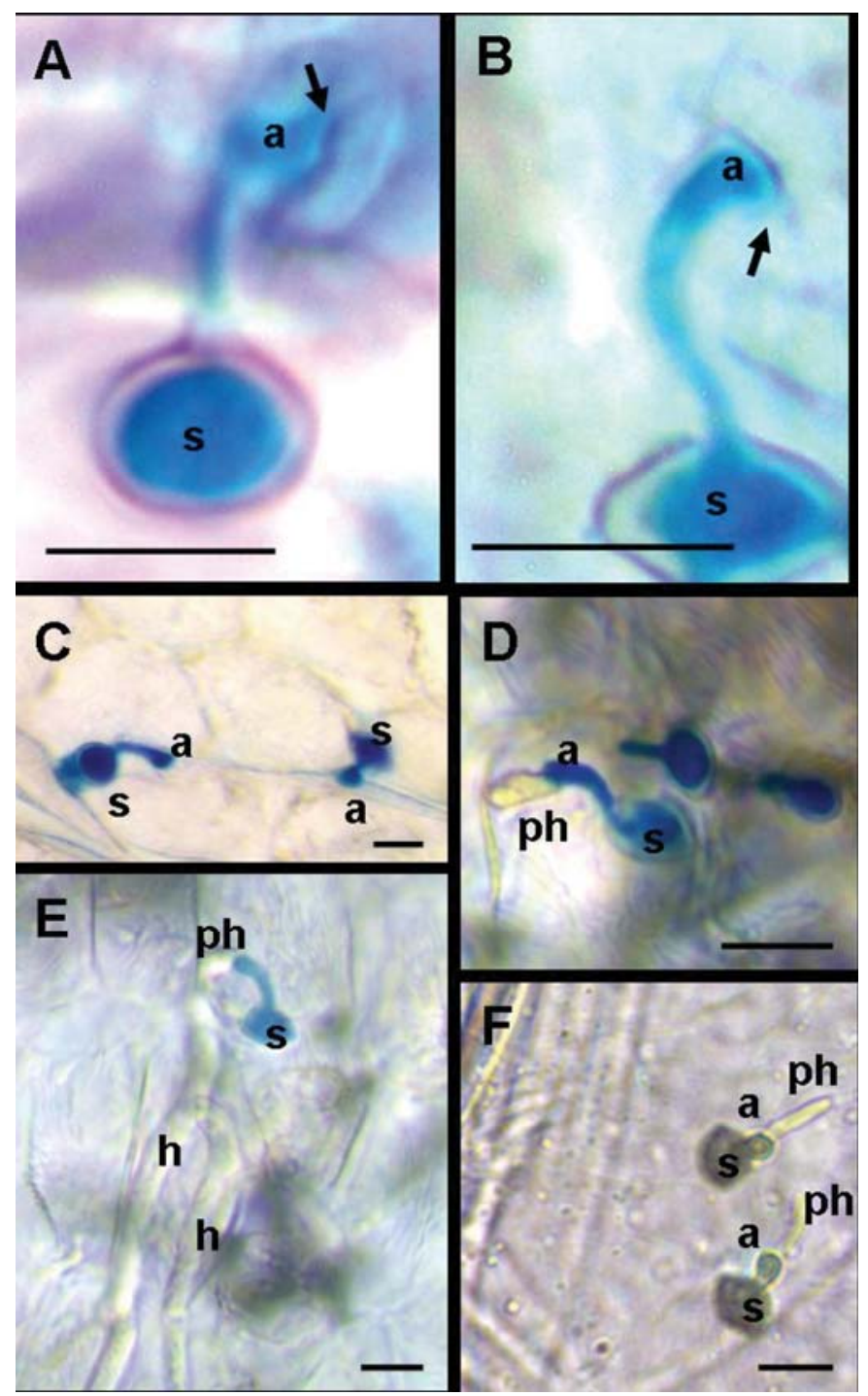

Fig. 3. Penetration of Prunus sp. and onion epidermal layers by Monilinia fructicola. A and B, Appressorium penetrates stage II nectarine fruit (cv. Fantasia) at $24 \mathrm{~h}$ postinoculation (hpi). Infection sites with depressed surfaces are indicated with arrows. C, D, and $\mathbf{E}$, Appressorium formation and penetration on almond flower petals. The inoculated petals are stained with lactophenolcotton blue. C, Appressoria form on almond petals at 4 hpi. D, Penetration can be observed at 9 hpi. A hyaline penetration hypha (ph), produced from the appressorium (a), can be observed growing under the epicuticular layer. E, At $24 \mathrm{hpi}$, the fungus has grown into the tissue. A hyaline penetration hypha growing under the epicuticular layer is indicated. F, M. fructicola produces appressoria and penetration hyphae on onion epidermal layers as observed by phase-contrast microscopy. Bar in each panel indicates $15 \mu \mathrm{m}$. s, spore; h, hypha. bation, in which one differentiated into an appressorium and the other into a long germ tube (Fig. 5). This also was observed occasionally on fruit surfaces. Collectively, these results suggest that components in $\mathrm{cV} 8$ juice broth and on the fruit surface stimulate appressoria to germinate into hyphae. Omission of $\mathrm{CaCO}_{3}$ from

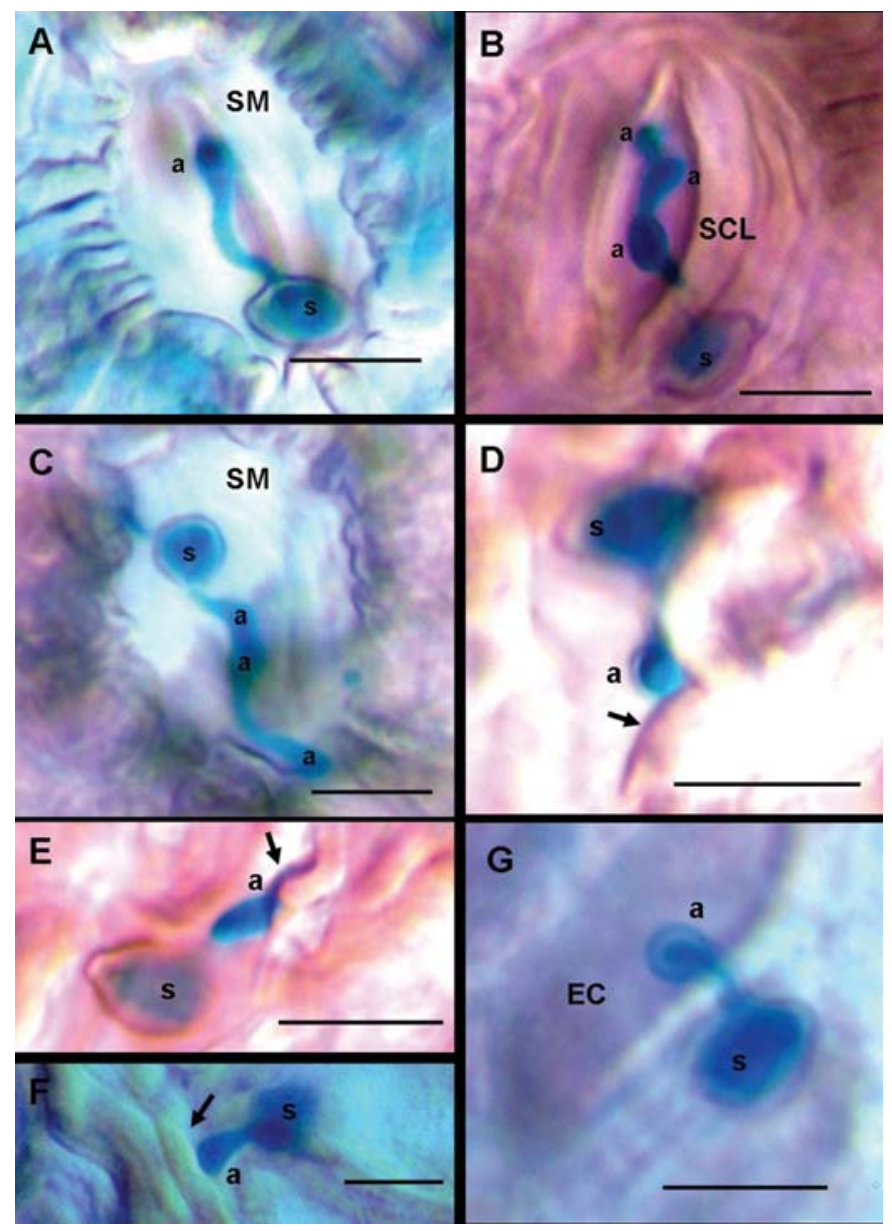

Fig. 4. Monilinia fructicola forms appressoria on stage II nectarine fruit in response to plant surface topography. $\mathbf{A}, \mathbf{B}$, and $\mathbf{C}$, Appressoria form on stomata of fruit. A and $\mathbf{B}$, One to three appressoria are produced on the stomatal guard cell lips from a conidium after sequential appressorial germinations. C, Conidia germinate toward lateral cells and produce appressoria on lateral cell grooves, even when the conidia initially lay on the stomata. D, E, $\mathbf{F}$, and $\mathbf{G}$, Appressoria form on the epicuticular layer of fruits. In $\mathbf{D}$ and $\mathbf{F}$, appressoria also appear to form on elevated structures (arrows). G, Appressorium formed on a concave surface. $\mathbf{A}, \mathbf{B}, \mathbf{D}$, and $\mathbf{G}$ are $\mathrm{cv}$. Fantasia. $\mathbf{C}$ is cv. Independence. Bar in each panel indicates $20 \mu \mathrm{m}$. s, spore; a, appressorium; h, hypha; SM, stoma; SCL, stomatal guard cell lip; EC, epidermal cell.

TABLE 1. Effect of washing the conidia of Monilinia fructicola on germination and appressorium formation on a polystyrene surface ${ }^{\mathrm{x}}$

\begin{tabular}{lcc}
\hline Treatments & $\begin{array}{c}\text { Conidial germination } \\
(\%)\end{array}$ & $\begin{array}{c}\text { Appressoria formed by } \\
\text { germlings }(\%)\end{array}$ \\
\hline Washed & $92 \mathrm{a}$ & $93 \mathrm{a}$ \\
Nontreated & $92 \mathrm{a}$ & $5 \mathrm{~b}$ \\
Washing control $^{\mathrm{y}}$ & $93 \mathrm{a}$ & $4 \mathrm{~b}$ \\
Original supernatant $^{\mathrm{z}}$ & $94 \mathrm{a}$ & $3 \mathrm{~b}$ \\
\hline
\end{tabular}

${ }^{\mathrm{x}}$ Data are from a single internally replicated experiment and are representative of three experiments with similar results. Each value is the mean of three separate determinations. Values followed by the same letter within a column are not significantly different $(\alpha=0.01)$ according to one-way analysis of variance using Tukey's test.

y Conidial suspensions that had been centrifuged repeatedly as washed conidia but no supernatants were removed or added.

${ }^{\mathrm{z}}$ The original supernatant removed after the first centrifugation was added back to the washed conidia, as described in Materials and Methods. 
$\mathrm{cV} 8$ juice broth did not significantly affect the frequency of appressorium formation and germination, as determined by regression analysis and paired $t$ test of the two slopes (Table 2). Appressorium formation also was sensitive to the concentration of conidia, with reduced frequency with increasing concentration of conidia (Table 3 ).

Hydrophobic surfaces induce appressorium formation. M. fructicola macroconidia formed appressoria on immature (late stage I and II) but not fully mature (late stage III) nectarine fruit. A major difference between these two developmental stages is the

TABLE 2. Effect of nutrients on germination and appressorium formation of washed conidia of Monilinia fructicola ${ }^{\mathrm{y}}$

\begin{tabular}{lcc}
\hline Treatments & $\begin{array}{c}\text { Conidial germination } \\
( \pm \mathrm{SD})(\%)\end{array}$ & $\begin{array}{c}\text { Appressoria formed by } \\
\text { germlings }( \pm \mathrm{SD})(\%)^{\mathrm{z}}\end{array}$ \\
\hline $\begin{array}{l}\text { Control (unwashed) } \\
\text { cV8 broth }\end{array}$ & $99 \pm 1$ & $13 \pm 1^{*}$ \\
0 & $97 \pm 2$ & $96 \pm 1$ \\
$0.01 \times$ & $99 \pm 1$ & $94 \pm 1$ \\
$0.1 \times$ & $100 \pm 0$ & $77 \pm 4$ \\
$1 \times$ & $100 \pm 0$ & $4 \pm 1^{*}$ \\
cV8 broth $\left(\right.$ no $\left.^{*} \mathrm{CaCO}_{3}\right)$ & $97 \pm 2$ & $96 \pm 1$ \\
0 & $99 \pm 0$ & $86 \pm 8$ \\
$0.01 \times$ & $99 \pm 1$ & $73 \pm 4$ \\
$0.1 \times$ & $99 \pm 1$ & $6 \pm 3^{*}$ \\
$1 \times$ & & \\
\hline
\end{tabular}

${ }^{\mathrm{y}}$ Ten-fold serial dilutions of clarified V8 $(\mathrm{cV} 8)$ juice broth or $\mathrm{cV} 8$ juice broth without $\mathrm{CaCO}_{3}$ were added to washed conidia to test the effect of the broth relative to the corresponding control ("0" broth) on appressorium formation on a polystyrene surface. Data are from a single trial that was representative of three trials. Each value is the mean and standard deviation (SD) of three replicates. Regression analysis using the square root of the fraction of the broth dilution showed that $\mathrm{cV} 8$ juice broth and $\mathrm{cV} 8$ juice broth containing $\mathrm{CaCO}_{3}$ significantly inhibited appressorium formation (cV8 juice broth, slope $=-97, P<0.0001 R^{2}=0.985 ; \mathrm{cV} 8$ juice broth plus $\mathrm{CaCO}_{3}$ slope $=$ $\left.-90, P<0.0001 R^{2}=0.983\right)$. A paired $t$ test indicated there was no significant difference between the slopes in the two broth treatments, indicating that the addition of $\mathrm{CaCO}_{3}$ had no effect on appressorium formation.

${ }^{\mathrm{z}}$ Asterisk indicates that there is no significant difference on percent appressorium formation between the broth treatment of washed spores and the unwashed control using multiple comparisons of means with the control by one-way analysis of variance using Dunnett's test.

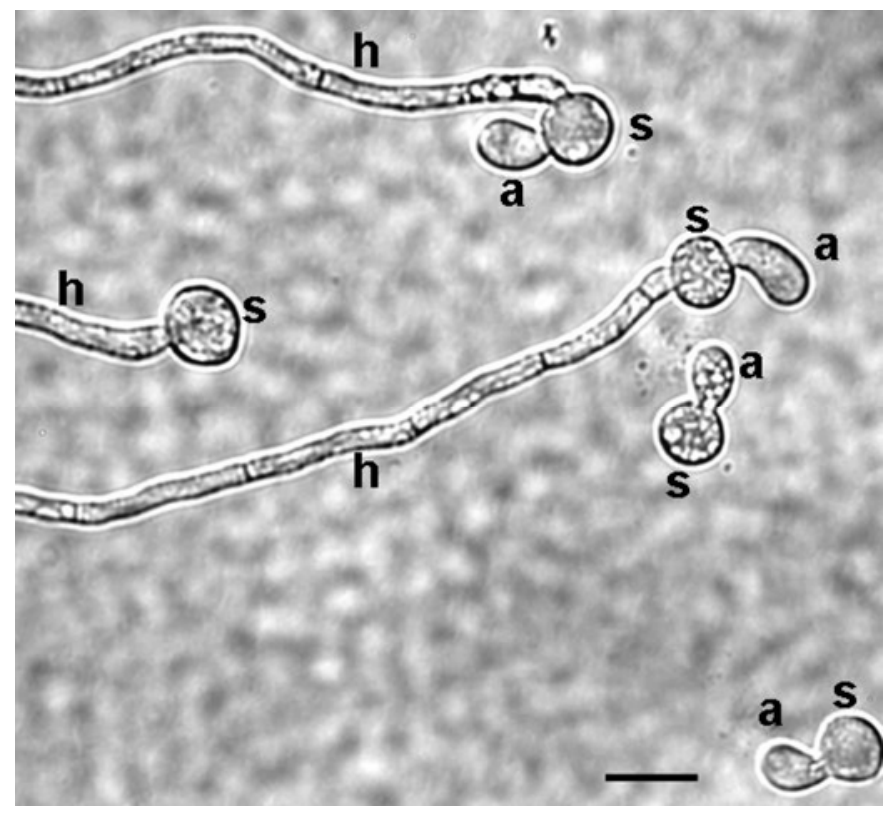

Fig. 5. Clarified V8 juice (cV8) induces secondary germination in Monilinia fructicola conidia on a polystyrene surface. Appressoria produced a second germ tube that grew into long hyphae after incubation in $1 \times \mathrm{cV} 8$ broth for $16 \mathrm{~h}$. Bar indicates $15 \mu \mathrm{m}$. s, spore; a, appressorium; h, hypha. hydrophobicity of the fruit surface. A drop that forms with rounder shape (i.e., a larger contact angle in relation to the surface) indicates that the surface has a more hydrophobic character (25). Based on this characterization, we observed that the surfaces of fruit at late stage I and stage II were more hydrophobic than fruit at early stage I and late stage III. Late stage III fruit surfaces were least hydrophobic, with the highest drop diameter (data not shown). Thus, frequency of appressorium formation appears to be related to hydrophobicity of the surfaces of fruit of different developmental stages.

To further test this hypothesis, washed conidia were placed on artificial hydrophobic and hydrophilic surfaces and evaluated for appressorium formation. A polystyrene petri dish, a parafilm ' $M$ ' membrane, and the hydrophobic side of Gel Bond, all strongly hydrophobic, resulted in $>85 \%$ of germinated conidia producing appressoria (Fig. 6). In contrast, $<50 \%$ of germinated conidia formed appressoria on glass slides and the hydrophilic surface of gel bond. On $2 \%$ agar, the most hydrophilic surface tested in our study, no appressoria formed. The hydrophobic effect on induction of M. fructicola appressoria was significant (Tukey's test; $P \leq$ $0.05)$.

$\mathrm{Ca}^{2+}$-calmodulin kinase- and cAMP-mediated signaling pathways are involved in $\boldsymbol{M}$. fructicola appressorium formation. Several pharmacological effectors were used to examine the possible signaling pathways involved in $M$. fructicola appressorium formation. PD98059, a MAP kinase inhibitor, inhibited germination, germ tube elongation and appressorium formation at $\geq 10 \mu \mathrm{M}$. At $5 \mu \mathrm{M}$, PD98059 inhibited germ tube elongation but did not significantly affect frequency of germination and appressorium formation (data not shown). cAMP significantly increased appres-

TABLE 3. Effect of conidial density of Monilinia fructicola on appressorium formation on a polystyrene surface

\begin{tabular}{lcc}
\hline Conidia $\left(\times 10^{3}\right)^{\mathrm{x}}$ & $\begin{array}{c}\text { Conidial germination }(\%) \\
( \pm \mathrm{SD})^{\mathrm{y}}\end{array}$ & $\begin{array}{c}\text { Appressoria formed by } \\
\text { germlings }(\%)^{\mathrm{z}}\end{array}$ \\
\hline 0.5 & $95 \pm 0$ & $93 \mathrm{a}$ \\
2 & $94 \pm 2$ & $39 \mathrm{~b}$ \\
5 & $94 \pm 2$ & $13 \mathrm{c}$ \\
\hline
\end{tabular}

${ }^{x}$ Number of washed conidia in each drop is indicated.

${ }^{y}$ Each value is the mean and standard deviation (SD) of three separate determinations.

${ }^{z}$ Values in the column with the same letter are not significantly different $(\alpha=$ 0.01 ) according to one-way analysis of variance using Tukey's test.

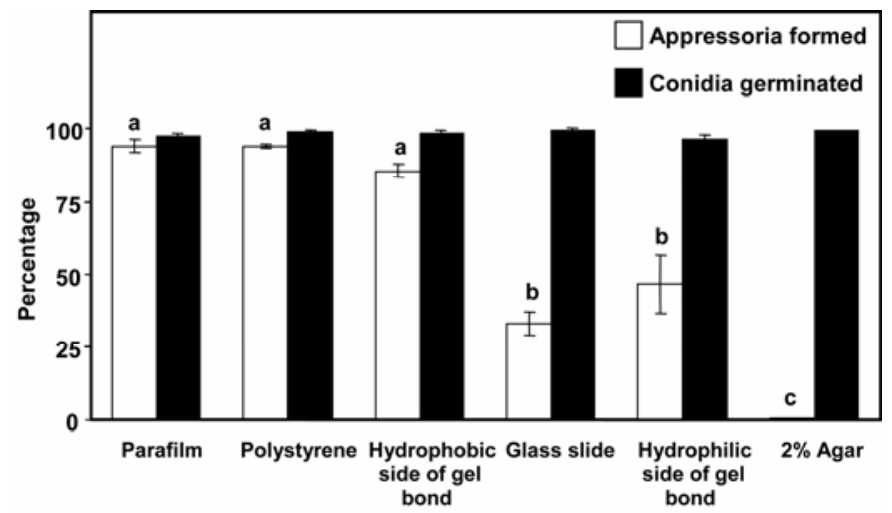

Fig. 6. The effect of surface hydrophobicity on Monilinia fructicola appressorium formation and conidial germination. Values represent the percentage germination of conidia and percentage of germinated conidia producing appressoria. The order of hydrophobicity among these materials is as follows: Parafilm $\mathrm{M} \geq$ polystyrene $\geq$ hydrophobic side of gel bond $>$ glass slide $>$ hydrophilic side of gel bond $>2 \%$ agar. Data represent the mean and standard deviation of three separate experiments for each treatment. Column values labeled with the same letter are not significantly different $(\alpha=0.05)$ according to Tukey's test. 
sorium formation of unwashed spores in a dose-dependent manner $(P<0.0001$ by regression analysis). Appressorium formation was increased from 8 to $60 \%$ at $10 \mathrm{mM}$ cAMP (Table 4). At this concentration, conidial germination frequency increased slightly and germ tube elongation was unchanged. However, at low concentrations ( 1 to $100 \mu \mathrm{M}$ ), cAMP inhibited appressorium formation on washed conidia but did not affect germination and germ tube elongation (Table 4). Although the inhibition of appressorium formation from washed conidia by the low concentration of cAMP was not dose dependent $(P>0.05$ by regression analysis), the inhibition for several concentrations of cAMP was significant when these were compared with the control by Dunnett's test $(P=0.05$ at 1 to $100 \mu \mathrm{M})$ (Table 4$)$. IBMX, a phosphodiesterase inhibitor, and $\mathrm{KN}-93$, a calcium/calmodulin kinase inhibitor, at 1 to $5 \mu \mathrm{M}$ concentrations had no effect on germination and germ tube elongation (Table 5). However, KN-93 at $10 \mu \mathrm{M}$ inhibited formation of appressoria from washed conidia by $90 \%$, and only slightly inhibited germination and germ tube elongation. IBMX showed a dose-dependent inhibition of appressorium formation at high concentrations (0.01 to $1 \mathrm{mM})$. Inhibition of conidial germination and germ tube elongation by IBMX was observed at $1 \mathrm{mM}$.

Appressorium formation is required for symptom development on Prunus spp. petals. $M$. fructicola used appressoria to penetrate Prunus spp. petals (Fig. 3C to E). To better assess the role of appressoria in $M$. fructicola pathogenicity, conidia mixed with KN-93 were inoculated onto flower petals. Necrotic lesions then were measured after $16 \mathrm{~h}$. KN-93 reduced appressorium formation and significantly reduced symptom development in a dose-dependent manner relative to the controls (Tables 6 and 7). Following inoculation with conidia in the presence of KN-93, the lesion areas that formed on petals from five Prunus spp. in four independent experiments were reduced compared with those formed in controls (Table 6). The reduction of lesion size by $\mathrm{KN}$ 93 appeared to be dose dependent. A concentration of $5 \mu \mathrm{M} \mathrm{KN}$ 93 significantly inhibited brown rot lesion development (paired $t$ test; $P<0.05$ ) (Table 7) but did not inhibit germination and germ tube elongation on petals (data not shown). These results support a requirement of appressoria by $M$. fructicola for infection and full symptom development in Prunus spp. petals.

TABLE 4. Effect of adenosine $3^{\prime}, 5^{\prime}$-cyclic monophosphate (cAMP) on germination and appressorium formation of unwashed and washed Monilinia fructicola conidia on a polystyrene surface

\begin{tabular}{|c|c|c|}
\hline $\begin{array}{l}\text { Treatment, } \\
\text { cAMP }(\mathrm{mM})^{\mathrm{x}}\end{array}$ & $\begin{array}{l}\text { Conidial germination (\%) } \\
\qquad \pm \mathrm{SD})^{\mathrm{y}}\end{array}$ & $\begin{array}{l}\text { Appressoria formed by } \\
\text { germlings }(\%)( \pm \mathrm{SD})^{\mathrm{y}, \mathrm{z}}\end{array}$ \\
\hline \multicolumn{3}{|l|}{ Unwashed } \\
\hline 0 & $91 \pm 0$ & $8 \pm 7$ \\
\hline 0.1 & $94 \pm 0$ & $8 \pm 4$ \\
\hline 1 & $98 \pm 0$ & $30 \pm 4$ \\
\hline 10 & $99 \pm 0$ & $60 \pm 8$ \\
\hline \multicolumn{3}{|l|}{ Washed } \\
\hline 0 & $98 \pm 1$ & $93 \pm 4$ \\
\hline 0.001 & $97 \pm 1$ & $76 \pm 6^{*}$ \\
\hline 0.01 & $96 \pm 1$ & $69 \pm 7 *$ \\
\hline 0.1 & $96 \pm 2$ & $81 \pm 7^{*}$ \\
\hline 1 & $98 \pm 2$ & $94 \pm 2$ \\
\hline
\end{tabular}

${ }^{x}$ cAMP was added to the final concentrations indicated to washed or unwashed conidial suspensions.

${ }^{y}$ Data are from a single trial that was representative of three trials. Each value is the mean and standard deviation (SD) of three separate replicates.

${ }^{\mathrm{z}}$ Regression analysis using the square root of the concentration of cAMP showed that cAMP significantly inhibited appressorium formation of unwashed conidia (slope $=17, P<0.0001, R^{2}=0.93$ ), but has no significant effect on appressorium formation (slope $=13, P=0.08, R^{2}=0.21$ ) of washed conidia. One-way analysis of variance using multiple comparisons of means with the control (without cAMP) using Dunnett's test showed that cAMP significantly inhibited appressorium formation from washed conidia at several concentrations (indicated by asterisk, $P=0.05$ ).

\section{DISCUSSION}

Appressorium formation in $M$. fructicola. In this study, we found that macroconidia of $M$. fructicola produce appressoria on artificial surfaces similar to appressoria observed in planta. Furthermore, appressorial formation on fruit surfaces depends to a large degree on fruit developmental stage. Conidia collected from infected fruit also were capable of producing appressoria on artificial surfaces. The simple appressoria, described previously on apricot fruit and cherry plum petals (9), were produced on stage II nectarine fruit and by washed macroconidia on all artificial hydrophobic surfaces tested in our study. M. fructicola produced appressoria on inductive surfaces (e.g., polystyrene) soon after conidial germination with a timing similar to that of the melanized appressoria produced by Colletotrichum spp. (13). Unlike Colletotrichum spp., M. fructicola appressoria were not or only lightly melanized.

The experiments with washed macroconidia also demonstrate that $M$. fructicola conidial preparations contain inhibitors of appressorium formation. Most likely this is due to nutrients carried over from the cultures, as evidenced from the study using serially diluted media. Similar phenomena also have been reported in Magnaporthe grisea (15). Although components in the cV8 juice broth inhibited the formation of Monilinia fructicola appressoria, the cV8 juice broth also could induce germination of already formed appressoria and promoted secondary germinations of conidia resulting in long germ tubes.

M. fructicola is a necrotrophic pathogen. One interpretation of our results is that when nutrients are abundant and accessible in the environment, as mimicked by culture media, M. fructicola predominantly exhibits a saprobic lifestyle rather than a pathogenic lifestyle, which is manifested in part by formation of specialized infection structures such as appressoria. This may explain, in part, why $M$. fructicola does not form appressoria on mature nectarine fruit, where nutrients are perhaps more readily accessible than on immature fruit.

Induction of M. fructicola appressoria. A wide variety of physical and chemical signals have been shown to influence de-

TABLE 5. Effects of the phosphodiesterase inhibitor 3-isobutyl-1-methylxanthine (IBMX) and the $\mathrm{Ca}^{2+} /$ calmodulin kinase inhibitor $\mathrm{KN}-93$ on Monilinia fructicola conidial germination and appressorium formation on a polystyrene surface

\begin{tabular}{lcc}
\hline $\begin{array}{l}\text { Treatment, } \\
\text { concentration }(\mu \mathrm{M})^{\mathrm{x}}\end{array}$ & $\begin{array}{c}\text { Conidial germination }(\%) \\
( \pm \mathrm{SD})^{\mathrm{y}}\end{array}$ & $\begin{array}{c}\text { Appressoria formed by } \\
\text { germlings }(\%)( \pm \mathrm{SD})^{\mathrm{z}}\end{array}$ \\
\hline IBMX & & $94 \pm 3$ \\
0 & $99 \pm 0$ & $87 \pm 5$ \\
0.1 & $97 \pm 2$ & $84 \pm 5$ \\
1 & $98 \pm 0$ & $81 \pm 8$ \\
10 & $97 \pm 4$ & $36 \pm 10$ \\
100 & $90 \pm 3$ & \\
$\mathrm{KN}-93$ & & $96 \pm 2$ \\
0 & $99 \pm 1$ & $88 \pm 5$ \\
0.01 & $97 \pm 0$ & $85 \pm 1$ \\
0.1 & $97 \pm 2$ & $78 \pm 9$ \\
1 & $99 \pm 1$ & $2 \pm 1$ \\
10 & $87 \pm 2$ & \begin{tabular}{c} 
\\
\hline
\end{tabular}
\end{tabular}

${ }^{x}$ IBMX and KN-93 were added to suspensions of washed conidia to achieve the final concentrations indicated. Data are from one individual experiment and are representative of three individual experiments with similar results.

${ }^{y}$ Each value is the mean and standard deviation (SD) of three separate replicates. Regression analysis showed significance with respect to the inhibition of conidial germination in the IBMX $(b=-0.078, P<0.0001)$ and KN-93 (b $=-1.05, P<0.0001)$ treatments.

${ }^{\mathrm{z}}$ Each value is the mean and SD of three separate replicates. Regression analysis showed significance with respect to the inhibition of appressorium formation in the IBMX $(\mathrm{b}=-0.52, P<0.0001)$ and $\mathrm{KN}-93(\mathrm{~b}=-8.78, P<0.0001)$ treatments. 
velopment of appressoria in phytopathogenic fungi. Based on our studies, surface features such as topography and hydrophobicity as well as chemical factors such as nutrients and fruit volatiles all have some influence on appressorium formation in $M$. fructicola. On fruit surfaces, M. fructicola produced appressoria on the stomatal guard cell lips and grooves adjacent to them, such as those formed at lateral cell junctions. Germ tubes apparently perceive particular topographical features, triggering differentiation of appressoria. Similar surface sensing has been reported in other phytopathogenic fungi, especially in $U$. appendiculatus on bean leaf stomata (16) and $C$. graminicola in relation to the grooves formed by anticlinal wall junctions (24).

In the absence of self-inhibitors and exogenous nutrients, surface hydrophobicity appears to be a key factor for triggering formation of $M$. fructicola appressoria. However, other regulatory signals cannot be ruled out due to the complexity of the physical and chemical environment of plant surfaces. On nectarine fruit, the frequency of appressoria varied during fruit development, indicating that additional fruit factors were involved in regulating appressorium formation. In an initial experiment, we tested volatiles emanating from different stage nectarine and peach fruit. The results showed volatiles from early stage I fruit and stage II fruit inhibited appressorium formation and conidial germination by $\approx 50 \%$, whereas volatiles from late stage III fruit enhanced conidial germination and germ tube elongation but inhibited appressorium formation of almost all germinated conidia.

On nectarine, germinated conidia rarely produced simple appressoria on fruit $<1.8 \mathrm{~cm}$ in diameter (early stage I), likely due to a higher density of stomata and lower hydrophobic character of the surface of fruit at this stage of development (data not shown). Upon reaching stage II, fruit have a lower stomatal density and a more hydrophobic surface (19). Thus, most conidia produced ap- pressoria on these fruit. The high densities of stomata on the stage I fruit also might result in a higher localized concentration of volatiles and exudates involved in inhibiting appressorial development. Mature fruit (late stage III) have a lower stomatal density and reduced hydrophobicity, with numerous cuticular cracks and fissures. Long germ tubes often were associated with these areas (Fig. 2D). Collectively, these observations are consistent with a role for both volatiles and surface hydrophobic character as important factors influencing appressorium development in $\mathrm{M}$. fructicola on early stage I and late stage III fruit, whereas hydrophobicity may be the overriding factor influencing development on stage II fruit. The nature of the specific fruit volatiles or chemicals that may be involved in stimulating or suppressing appressoria development needs further study, especially because the mix of volatile components varies among different fruit stages (35).

The signal transduction pathways involved in appressorium formation have been investigated in Magnaporthe grisea, Colletotrichum spp., and a few other fungi $(11,12,32)$. In this study, we primarily investigated signal pathways that regulate appressorium development using pharmacological effectors. These effectors can have multiple effects on fungal development, depending on the dosage used. In our experiments, the pharmacological effectors influenced germination, appressorium formation, and germ tube elongation at certain concentrations. The addition of cAMP induces appressorium formation in some fungi and there may be an optimal concentration, depending on the species, at which cAMP exerts a stimulatory effect $(3,22,25)$. For example, in B. graminis f. sp. hordei, the level of intracellular cAMP is very low prior to appressorium formation (e.g., attomole/spore) (22). The addition of low concentrations $(1 \mu \mathrm{M})$ of cAMP will stimulate appressorium development in this fungus, but moderate

TABLE 6. Reduction of brown rot lesion development on flower petals following inoculation in the presence of the Ca ${ }^{2+} / \mathrm{calmodulin}^{\mathrm{k} i n a s e}$ inhibitor $\mathrm{KN}-93^{\mathrm{y}}$

\begin{tabular}{|c|c|c|c|c|c|}
\hline \multirow[b]{2}{*}{ Species, cultivar } & \multirow[b]{2}{*}{ Conidia/droplet } & \multirow[b]{2}{*}{$n$} & \multicolumn{3}{|c|}{ Reduction of lesion size $(\%)$ in presence of $\mathrm{KN}-93(\mu \mathrm{M})^{\mathrm{z}}$} \\
\hline & & & 10 & 5 & 2.5 \\
\hline \multicolumn{6}{|c|}{ Prunus dulcis (almond) } \\
\hline Butte & 250 & 35 & 87 & 80 & 77 \\
\hline \multicolumn{6}{|c|}{ P. persicae var. nectarina (nectarine) } \\
\hline Independence & 250 & $37-42$ & 59 & 49 & 22 \\
\hline Flavor Top & 500 & 22 & 100 & 93 & 41 \\
\hline Flavor Top & 250 & 25 & 100 & 89 & 67 \\
\hline Fantasia & 250 & $11-21$ & 100 & 91 & 48 \\
\hline \multicolumn{6}{|c|}{ P. persicae (peach) } \\
\hline Carson & 250 & $21-24$ & 95 & 85 & 53 \\
\hline
\end{tabular}

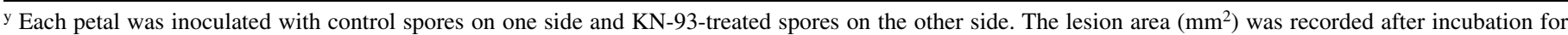
12 to $16 \mathrm{~h}$. The number of blossoms $(n)$ evaluated for each cultivar or species is indicated. In some cases, a range is given because sample sizes were unequal for the different KN-93 concentrations for that cultivar.

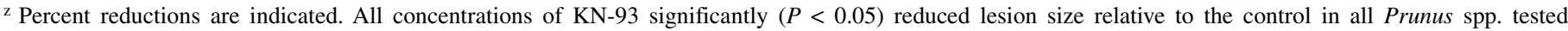
according to paired $t$ test of actual lesion size of inoculated petals (not shown).

TABLE 7. Reduction of brown rot lesion size on flower petals by KN-93

\begin{tabular}{|c|c|c|c|c|c|}
\hline \multirow[b]{2}{*}{ Species, cultivar } & \multirow[b]{2}{*}{ Conidia/droplet } & \multirow[b]{2}{*}{$n$} & \multicolumn{3}{|c|}{ Lesion size in $\mathrm{mm}^{2} /($ no. of petals with lesions) } \\
\hline & & & Control $^{\mathrm{y}}$ & $\mathrm{KN}-93^{\mathrm{y}}$ & $P$ value ${ }^{\mathrm{z}}$ \\
\hline \multicolumn{6}{|c|}{ Prunus dulcis (almond) } \\
\hline Butte & 250 & 35 & $12.8 /(21)$ & $2.6 /(6)$ & $<0.001$ \\
\hline \multicolumn{6}{|c|}{ P. persicae var. nectarina (nectarine) } \\
\hline Independence & 250 & 38 & $9.1 /(25)$ & $4.7 /(17)$ & $<0.01$ \\
\hline Flavor Top & 500 & 23 & $9.0 /(10)$ & $0.2 /(1)$ & $<0.001$ \\
\hline Flavor Top & 250 & 25 & $8.4 /(15)$ & $0.9 /(2)$ & $<0.001$ \\
\hline Fantasia & 250 & 21 & $5.5 /(11)$ & $0.5 /(2)$ & $<0.001$ \\
\hline \multicolumn{6}{|c|}{ P. persicae (peach) } \\
\hline Carson & 250 & 21 & $2.9 /(5)$ & $0.4 /(1)$ & $<0.05$ \\
\hline
\end{tabular}

${ }^{\mathrm{x}}$ Data analyzed and presented in this table are from Table $6 ; 5 \mu \mathrm{M}$ of KN-93 was used; $n$ indicates number of blossoms evaluated.

${ }^{y}$ Mean lesion areas $\left(\mathrm{mm}^{2}\right)$ and the number of inoculated petals with brown rot lesions are presented.

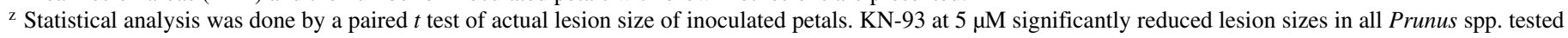
$(P<0.05)$. 
concentrations (10 to $100 \mu \mathrm{M}$ ) have no effect. In M. grisea, however, appressorium development on a noninductive surface requires concentrations of cAMP as high as $10 \mathrm{mM}$ (25). Similarly, we found that $10 \mathrm{mM}$ cAMP overcomes any inhibitory effect of nutrients and self-inhibitors on the development of appressoria by Monilinia fructicola.

The addition of low to moderate ( 1 to $100 \mu \mathrm{M})$ concentrations of cAMP inhibited appressorium formation of washed conidia of M. fructicola without inhibiting germination. Paradoxically, the cyclic nucleotide phosphodiesterase inhibitor IBMX, which is presumed to increase intracellular cAMP levels, also inhibited appressorium formation of washed conidia without inhibiting germination. To reconcile these results, it is conceivable that IBMX elevates intracellular cAMP concentrations to levels similar to those achieved by exogenous treatment of conidia with 1 to $100 \mu \mathrm{M}$ cAMP. Thus, in the absence of other inhibitory factors (e.g., nutrients), low concentrations of cAMP seem to arrest development of M. fructicola appressoria. This inhibition, whether imposed by nutrients, self-inhibitors, or low intracellular levels of cAMP, can be overcome by treatment with high concentrations of cAMP.

Our results support the notion that cAMP homeostasis plays a critical role in differentiation of appressoria from germinated conidia in M. fructicola. In addition, the involvement of a calcium/ calmodulin kinase-regulated pathway in appressorium development is suggested by the inhibitory effect of KN-93

The possible function of $M$. fructicola appressoria. Although morphology and the degree of differentiation of appressoria vary significantly among fungal species, it generally is accepted that these structures mediate penetration. The mechanisms are diverse, because appressorium-mediated penetration includes entry via natural openings (stomata) as well as aggressive direct penetration of intact cuticle layers. Here, we show that $M$. fructicola directly penetrates Prunus spp. petals, green nectarine fruit, and onion epidermal cells through penetration pegs produced from appressoria (Figs. 3 and 4). Furthermore, KN-93, which inhibited M. fructicola appressorium formation but not germination and germ tube elongation, inhibited lesion development on petals from several Prunus spp. when it was mixed with the inoculum. The reduction of disease was highly dependent on the concentration of KN-93. The results strongly support a role for $M$. fructicola appressoria in disease development on petals and these appressoria most likely function in penetration during infection of immature tissues.

Early infections of immature fruit by $M$. fructicola often remain quiescent, but these can reactivate upon fruit maturity $(4,10$, $18,26,36)$. In our study, we found that $M$. fructicola produces appressoria and maintains these structures on stage II fruit even after 1 week of incubation. Infected areas appear as small spots that become yellow or water soaked but do not develop into lesions. It is possible that the appressoria with penetration pegs also function as resting structures on immature fruit.

In conclusion, appressoria produced on artificial and plant surfaces by $M$. fructicola were very similar in appearance, and their formation depended on fruit developmental stage. External effectors and regulatory signals described for appressorial development in other fungi also were found to influence appressorium development in $M$. fructicola. The seasonal availability of host materials such as Prunus spp. blossoms and fruit complicates the experimental study of pathogenicity and appressorial development in this system; however, the artificial inductive surfaces described herein could be useful in future studies to understand aspects of the regulatory mechanisms governing differentiation of appressoria in this host-pathogen interaction. Careful study of quantitative differences in the active components present in fruit of different developmental stages may well provide new insights into the infection process, quiescence, and brown rot disease development.

\section{ACKNOWLEDGMENTS}

This research was supported in part by grants from the California Cling Peach Board and the US-Israel BARD program. We thank K.-R. Chung, L. Epstein, T. Gordon, and J. Labavitch for their critical review of the manuscript; and S. Marek and P. Weyman for their helpful discussions.

\section{LITERATURE CITED}

1. Aist, J. R., and Bushnell, W. R. 1991. Invasion of plants by powdery mildew fungi, and cellular mechanisms of resistance. Pages 321-345 in: The Fungal Spore and Disease Initiation in Plants and Animals. G. T. Cole and H. C. Hoch, eds. Plenum Press, New York.

2. Balhadere, P. V., and Talbot, N. J. 2001. Pde1 encodes a p-type ATPase involved in appressorium-mediated plant infection by the rice blast fungus Magnaporthe grisea. Plant Cell 13:1987-2004.

3. Barhoom, S., and Sharon, A. 2004. cAMP regulation of "pathogenic" and "saprophytic" fungal spore germination. Fungal Genet. Biol. 41:317-326.

4. Biggs, A. R., and Northover, J. 1988. Early and late-season susceptibility of peach fruits to Monilinia fructicola. Plant Dis. 72:1070-1074.

5. Bircher, U., and Hohl, H. R. 1997. Environmental signalling during induction of appressorium formation in Phytophthora. Mycol. Res. 101:395-402.

6. Boehm, E. W. A., Ma, Z., and Michailides, T. J. 2001. Species-specific detection of Monilinia fructicola from California stone fruits and flowers. Phytopathology 91:428-439.

7. Bostock, R. M., Wilcox, S. M., Wang, G., and Adaskaveg, J. E. 1999. Suppression of Monilinia fructicola cutinase production by peach fruit surface phenolic acids. Physiol. Mol. Plant Pathol. 54:37-50.

8. Clay, R. P., Enkerli, J., and Fuller, M. S. 1994. Induction and formation of Cochliobolus sativus appressoria. Protoplasma 178:34-47.

9. Cruickshank, R. H., and Wade, G. C. 1992. Production of appressoria by Monilinia fructicola. Mycol. Res. 96:425-428.

10. Cruickshank, R. H., and Wade, G. C. 1992. The activation of latent infections of Monilinia fructicola on apricots by volatiles from the ripening fruit. J. Phytopathol. 136:107-112.

11. Dean, R. A. 1997. Signal pathways and appressorium morphogenesis. Annu. Rev. Phytopathol. 35:211-234.

12. Deising, H. B., Werner, S., and Wernitz, M. 2000. The role of fungal appressoria in plant infection. Microbes Infect. 2:1631-1641.

13. Flaishman, M. A., and Kolattukudy, P. E. 1994. Timing of fungal invasion using host's ripening hormone as a signal. Proc. Natl. Acad. Sci. USA 91:6579-6583.

14. Hajek, A. E., Filotas, M. J., and Ewing, D. C. 2002. Formation of appressoria by two species of lepidopteran-pathogenic Entomophthorales. Can. J. Bot. 80:220-225.

15. Hegde, Y., and Kolattukudy, P. E. 1997. Cuticular waxes relieve selfinhibition of germination and appressorium formation by the conidia of Magnaporthe grisea. Physiol. Mol. Plant Pathol. 51:75-84.

16. Hoch, H. C., Staples, R. C., Whitehead, B., Comeau, J., and Wolf, E. D. 1987. Signaling for growth orientation and cell differentiation by surface topography in Uromyces. Science 235:1659-1662.

17. Howard, R. J., Ferrari, M. A., Roach, D. H., and Money, N. P. 1991. Penetration of hard substrates by a fungus employing enormous turgor pressures. Proc. Natl. Acad. Sci. USA 88:11281-11284.

18. Ibbotson-Darhower, H., Hickey, K. D., and Travis, J. W. 1998. Susceptibility of peach and nectarine fruit at different developmental stages to Monilinia fructicola. (Abstr.) Phytopathology 88:S130.

19. Ishida, M., Hirata, H., Kitajima, A., and Sobajima, Y. 1990. Development and density of stomata on fruit surfaces during fruit growth in nectarine. J. Jpn. Soc. Hortic. Sci. 58:793-800.

20. Kim, Y.-K., Kawano, T., Li, D., and Kolattukudy, P. E. 2000. A mitogenactivated protein kinase kinase required for induction of cytokinesis and appressorium formation by host signals in the conidia of Colletotrichum gloeosporioides. Plant Cell 12:1331-1343.

21. Kim, Y.-K., Li, D., and Kolattukudy, P. E. 1998. Induction of $\mathrm{Ca}^{2+}$ calmodulin signaling by hard-surface contact primes Colletotrichum gloeosporioides conidia to germinate and form appressoria. J. Bacteriol. 180:5144-5150.

22. Kinane, J., Dalvin, S., Bindslev, L., Hall, A., Gurr, S., and Oliver, R. 2000. Evidence that the cAMP pathway controls emergence of both primary and appressorial germ tubes of barley powdery mildew. Mol. Plant-Microbe Interact. 13:494-502.

23. Kinane, J., and Oliver, R. P. 2003. Evidence that the appressorial development in barley powdery mildew is controlled by MAP kinase activity in conjunction with the cAMP pathway. Fungal Genet. Biol. 39:94-102.

24. Lapp, M. S., and Skoropad, W. P. 1978. Location of appressoria of Colletotrichum graminicola on natural and artificial barley leaf surfaces. Trans. Br. Mycol. Soc. 70:225-228. 
25. Lee, Y.-H., and Dean, R. A. 1993. cAMP regulates infection structure formation in the plant pathogenic fungus Magnaporthe grisea. Plant Cell 5:693-700.

26. Luo, Y., and Michailides, T. J. 2001. Factors affecting latent infection of prune fruit by Monilinia fructicola. Phytopathology 91:864-872.

27. Mendgen, K., and Deising, H. 1993. Tansley review no. 48: Infection structures of fungal plant pathogens: A cytological and physiological evaluation. New Phytol. 124:192-213.

28. Mendgen, K., Hahn, M., and Deising, H. 1996. Morphogenesis and mechanisms of penetration by plant pathogenic fungi. Annu. Rev. Phytopathol. 34:367-386.

29. Ogawa, J. M., Zeha, E. I., Bird, G. W., Ritchie, D. F., Uriu, K., and Uyemoto, J. K. 1995. Compendium of Stone Fruit Diseases. American Phytopathological Society Press, St. Paul, MN.

30. Prusky, D. 1996. Pathogen quiescence in postharvest diseases. Annu. Rev. Phytopathol. 34:413-434.

31. Pryce-Jones, E., Carver, T., and Gurr, S. J. 1999. The roles of cellulase enzymes and mechanical force in host penetration by Erysiphe graminis $\mathrm{f}$. sp. hordei. Physiol. Mol. Plant Pathol. 55:175-182.

32. Talbot, N. J. 2003. On the trail of a cereal killer: Exploring the biology of Magnaporthe grisea. Annu. Rev. Microbiol. 57:177-202.

33. Terhune, B. T., and Hoch, H. C. 1993. Substrate hydrophobicity and adhesion of Uromyces urediospores and germlings. Exp. Mycol. 17:241252.

34. Tucker, S. L., and Talbot, N. J. 2001. Surface attachment and prepenetration stage development by plant pathogenic fungi. Annu. Rev. Phytopathol. 39:385-417.

35. Visai, C., and Vanoli, M. 1997. Volatile compound production during growth and ripening of peaches and nectarines. Sci. Hortic. 70:15-24.

36. Wade, G. C., and Cruickshank, R. H. 1992. The establishment and structure of latent infections with Monilinia fructicola on apricots. J. Phytopathol. 136:95-106.

37. Warwar, V., Oved, S., and Dickman, M. B. 2000. Antisense expression of the calmodulin gene from Colletotrichum trifolii impairs prepenetration development. FEMS Microbiol. Lett. 191:213-219.

38. Wynn, W. K. 1976. Appressorium formation over stomates by the bean rust fungus in response to a surface contact stimulus. Phytopathology 66:136-146.

39. Yang, Z., and Dickman, M. B. 1999. Molecular cloning and characterization of $C t-P K A R$, a gene encoding the regulatory subunit of cAMPdependent protein kinase in Colletotrichum trifolii. Arch. Microbiol. 171:249-256.

40. Zucconi, F. 1986. Peach. Pages 303-321 in: CRC Handbook of Fruit Set and Development. S. P. Monselise, ed. CRC Press, Inc., Boca Raton, FL. 